\title{
Risk-Based Dynamic Anisotropic Operational Safety Bound for Rotary UAV Traffic Control
}

\author{
Jueming $\mathrm{Hu}^{1}$, Heinz Erzberger ${ }^{2}$, Kai Goebel ${ }^{3}$, and Yongming $\mathrm{Liu}^{4}$ \\ ${ }^{1,4}$ Arizona State University, Tempe, AZ, 85281 \\ jueming.hu@asu.edu \\ yongming.liu@asu.edu \\ ${ }^{2}$ University of California, Santa Cruz, 95064 \\ heinz.erzberger@nasa.gov \\ ${ }^{3}$ PARC, Palo Alto, CA, 94304 \\ Luleå Technical University, Luleå, Sweden \\ kai.goebel@ltu.se
}

\begin{abstract}
This paper proposed a novel method to determine probabilistic operational safety bound for unmanned aircraft traffic management. The key idea is to implement probabilistic uncertainty quantification and design the operational safety bound shape considering UAV's heading direction. Operational safety bound is used to identify a virtual geographic boundary to protect aircraft and to ensure airspace safety. The proposed operational safety bound is calculated as a function of vehicle performance characteristics, state of vehicle, weather and other probabilistic parameters that affect the real position of vehicle such as position error from the Global Positioning System (GPS). It is calculated individually for each vehicle using real-time data and probability simulation. It considers the heading direction of vehicle and thus it is an anisotropic design. Monte Carlo simulations are conducted to estimate the operational safety bound size with a specified probability of failure. Results indicate that uncertainty is crucial for the operational safety bound's size. Sensitivity study shows that UAV speed has the largest effect on the operational safety bound size. Analysis of impact of failure probability shows that operational safety bound size increases with the decrease in allowable failure probability, but the bound size based on different operational safety bound concept increases at different rate.
\end{abstract}

\footnotetext{
Jueming $\mathrm{Hu}$ et al. This is an open-access article distributed under the terms of the Creative Commons Attribution 3.0 United States License, which permits unrestricted use, distribution, and reproduction in any medium, provided the original author and source are credited.
}

\section{INTRODUCTION}

An unmanned aerial vehicle (UAV) is an aircraft which operates without a human pilot aboard (International Civil Aviation Organization, 2011). The Unmanned Aircraft System (UAS) consists of many UAVs, communication links, and controllers. Careful separation assurance between UAVs is crucial to ensure the safety of UAS (Federal Aviation Administration, 2016). Small UAS has been applied to many areas, such as surveillance, deliveries, search and rescue, traffic monitoring, videography, and precision agriculture. Some operations - such as urban air mobility are supposed to occur in areas close to buildings or airports. NASA initiated UAS Traffic Management (UTM) research that could enable large-scale low altitude UAS operations (Kopardekar et al., 2016).

Among many aspects for fast growth of UTM research, safety and efficiency are the most important factors that are critical for the large deployment of this concept. Collision avoidance, including collisions with UAVs, buildings, and other obstacles is one major safety problem in UAS traffic. The concept of operational safety bound has been studied in recent years. It is used to demark a virtual geographic boundary that limits where an aircraft is allowed to fly. It has also emerged as a way to manage trajectory separation (D'Souza, Ishihara, Nikaido, \& Hasseeb, 2016). Obviously, larger trajectory separation means that the airspace can hold fewer UAVs. Maximizing airspace capacity while minimizing safe aircraft separation plays an important role in balancing safety and efficiency for UAS traffic control and management.

There are two basic types of aircraft safety bounds: static operational safety bound (i.e., operational safety boundary is 
predetermined and does not change with UAV operation conditions) and dynamic operational safety bound (i.e., operational safety boundary changes according to UAV operation conditions) (Miraglia \& Hook, 2017). The near mid-air collision (NMAC) threshold for a conflict between commercial aircrafts is well-defined for ATM (Administration, 2011), but there has not been a standard value for UAV yet. Ong and Kochenderfer (Ong \& Kochenderfer, 2017) defined an NMAC to be $30 \mathrm{~m}$ between two drones. The threshold of $30 \mathrm{~m}$ was obtained from scaling the standard NMAC threshold for commercial aircraft by a reasonable scaling factor for smaller drones. The determination of the "scaling factor" appears not to be very rigorous. In (D'Souza et al., 2016) the term "geofence" is used. A geofence consists of distance buffers that enclose individual trajectories. In particular, a UTM prototype used a geofence size of 30 meters around a UAV trajectory horizontally and vertically (D'Souza et al., 2016). Bulusu et al. (Bulusu, Sengupta, \& Liu, 2016) performed simulations to estimate the UAS traffic density based on the NMAC threshold assumptions of 50, 100, 150, 200, 250, and 300 meters, respectively. Nikolos and Brintaki (Nikolos \& Brintaki, 2005) state that each UAV has to keep a safety distance from obstacles. Trajectories of UAVs in a maritime environment are planned with a safety distance set equal to $12.5 \%$ of the length along the rectangular terrain (Nikolos \& Brintaki, 2005). This follows the idea of operational safety bound for commercial aircrafts, which is at least $300 \mathrm{~m} \mathrm{(1000}$ ft) vertically below flight level (FL) 290 (ICAO, 2016). Weinert et al. (Weinert, Campbell, Vela, Schuldt, \& Kurucar, 2018) recommend that, for small UAVs weighing less than $55 \mathrm{lbs}$ operating at low altitudes, horizontal separation is $2000 \mathrm{ft}$ and vertical separation is $250 \mathrm{ft}$. Wu et al. (Wu et al., 2018) suggested horizontal separation of $0.36 \mathrm{nmi}$ and vertical separation of $450 \mathrm{ft}$ for small UAS (55 lbs vehicle or less) in low-altitude controlled airspace around airports. Bosson and Lauderdale (Bosson \& Lauderdale, 2018) suggested the operational safety bound of $0.3 \mathrm{nmi}$ horizontally and $100 \mathrm{ft}$ vertically for highly-autonomous AutoResolver algorithm in UAM management. AutoResolver is designed to handle conflicts predicted to occur in the range of approximately 2-20 min (Erzberger, Lauderdale, \& Chu, 2012). For large UAS in high-altitude airspace, Cook and Brooks (Cook \& Brooks, 2015) suggest a Horizontal Miss Distance (HMD) of $0.66 \mathrm{nmi}$ and the Vertical Miss Distance (VMD) of $450 \mathrm{ft}$. The abovementioned operational safety bound methods belong to the concept of "static operational safety bound". Compared to the relatively large number of studies for static operational safety bound, dynamic operational safety bound concept has not been thoroughly investigated. In (Paielli \& Erzberger, 2017) trajectory specification is conducted for aircraft by adding trajectory bound to guarantee safe separation. The bounding space is defined by cross-track, along-track, and vertical tolerances (Paielli \& Erzberger, 2017). The tolerances are dynamic and based on the aircraft navigation capabilities and the current traffic situation (Paielli \& Erzberger, 2017). D'Souza et al. (D'Souza et al., 2016) proposed a geofence concept which is also called 'keep-in' region that the UAV may fly to due to perturbation from wind. It varies around an unmanned aircraft operation based on vehicle performance and wind (D'Souza et al., 2016). There are two geofence sizes calculated for horizontal and vertical direction, respectively (D'Souza et al., 2016). The results of the study showed that the horizontal and vertical geofence sizes are reduced by approximately half compared to the concept of static geofence (e.g., $30 \mathrm{~m}$ ) (D'Souza et al., 2016). Moreover, research on artificial force field for haptic collision avoidance uses the concept similar to operational safety bound to determine the risk field around a UAV. The parametric risk field allows adjustments based on UAV operations (Lam, Boschloo, Mulder, \& Van Paassen, 2009; Brandt \& Colton, 2010).

A quick summary can be drawn based on the above brief review for the existing gaps in the operational safety bound determination in UTM. First, most existing separation distances are determined by experts' experiences or simple models. Many important factors, such as UAV performance, positioning and control parameters, and speeds are not explicitly included. Development of such a model is very valuable to evaluate the relationship between operation conditions with operational safety bound and the operational safety bound impact on traffic management. Next, UAV operation is stochastic in nature and randomness exists in almost every aspect of UTM. Inclusion of uncertainty quantification of operational safety bound is critical for the future safety analysis (e.g., probability of failure) (Liu \& Goebel, 2018; Pang, Yao, Hu, \& Liu, 2019; Wang, Pang, Liu, Dutta, \& Yang, 2019). Thus, a probabilistic risk-based operational safety bound methodology is more appropriate in this regard, which is the major motivation of the proposed study.

This paper proposes a novel method to determine operational safety bound for unmanned aircraft traffic management. The method includes measurements of UAV performance, positioning, control characteristics, and onboard flight tracking system. Considering the vehicle's heading direction results in an anisotropic vectorized separation boundary shape. Additionally, the proposed operational safety bound is probabilistic and includes various uncertainty sources. For example, errors on the Global Positioning System (GPS), wind magnitude and direction randomness, and UAV maximum deceleration are considered here.

The paper is organized as follows. First, the methodology of the proposed operational safety bound is introduced. A simple mechanism model for rotary-wing UAV collision avoidance is proposed together with a parametrized uncertainty quantification framework for probabilistic analysis. A one-dimensional case is illustrated to show the basic concept and the methodology is extended to a more 
general two-dimensional case. Results from Monte Carlo simulation studies are compared with different static and dynamic operational safety bound concepts. A sensitivity analysis is provided to show the important factors affecting the operational safety bound calculation. The impact of risk levels on the operational safety bound size is also discussed. Several conclusions are drawn, and future research directions are identified.

\section{Proposed Methodology}

In this study, we focus on rotary-wing UAV. The proposed methodology can be extended to other types of UAV, such as fixed-wing UAV or hybrid UAV. In this study, the UAV is assumed to be a point mass with speed and heading direction. It is assumed that UAV can decelerate to zero speed and hover. All flights are assumed to be at the same altitude and no vertical separation is considered in this study. The above assumptions are used consistently throughout this paper.

The key concept of the proposed methodology is based on the observation that most positioning systems, such as GPS, have certain updating frequency (e.g., updating for every second). In addition, the control system in UAV might have a further delay to use the updated positioning information. Thus, UAV may fly for a certain duration without precise positioning information. The proposed methodology suggests a new operational safety bound determination to minimize the collision risk during this time duration. The proposed operational safety bound is discussed in one-dimensional and two-dimensional cases separately. Details are shown below.

\subsection{Operational Safety Bound for One-Dimensional Case}

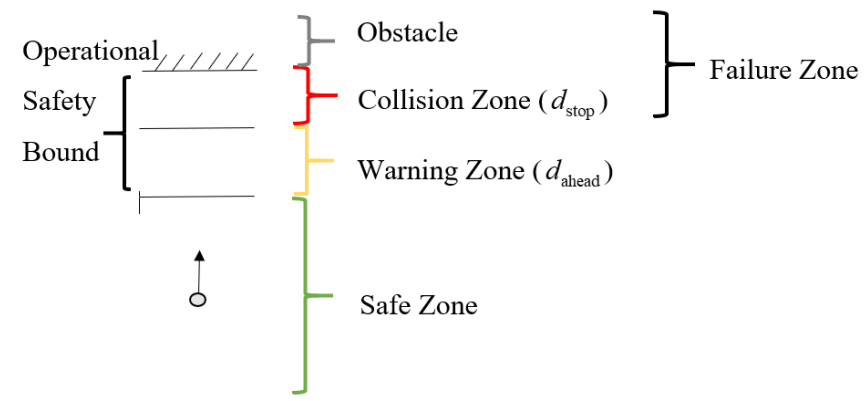

Figure 1. Concept of the proposed operational safety bound.

The proposed operational safety bound tries to prevent collision for a UAV which is under its normal operation (in Safe Zone in Figure 1). The operational safety bound is determined so that the UAV will not fly into the Failure Zone. The Failure Zone has two possible scenarios: 1) flying into the obstacle directly; 2) flying into the Collision Zone where the UAV's maximum deceleration cannot prevent the vehicle from hitting the obstacle. Between the Safe Zone and Failure Zone, there is a Warning Zone (see Figure 1). The physical meaning of the Warning Zone is that a UAV can have an updated positioning information (and corresponding control action implementation) before reaching the Collision Zone. Thus, corrective actions can be taken by the UAV to avoid the possible collision. In this sense, the Warning Zone can be considered as a "buffer" zone to provide sufficient time for UAV to mitigate collision risk. Thus, the proposed operational safety bound is defined as the distance between the obstacle and a UAV under normal operation. It includes two parts as shown in Figure 1: Collision Zone and Warning Zone.

Schematic illustration and symbols are shown in Figure 1 to better explain the proposed concept. Safe Zone, Warning Zone, and Collision Zone are highlighted using green, yellow, and red, respectively. This mimics the common traffic light for easy understanding. Mathematically, the Collision Zone size is expressed as

$$
d_{\text {stop }}=\frac{\left|\mathbf{V}_{\text {total }}\right|^{2}}{2(a)}
$$

where $d_{\text {stop }}$ is the minimum stopping distance for a UAV to decelerate until it hovers. $d_{\text {stop }}$ may be directly provided (DJI, 2018) or can be calculated by using the maximum deceleration rate $a$ for a UAV. $a$ is a critical performance metric and usually depends on the motor power, aircraft design, and weight of a rotary-wing UAV. $\mathbf{V}_{\text {total }}$ is the true ground velocity vector of the UAV and can be expressed as

$$
\mathbf{V}_{\text {total }}=\mathbf{V}_{\mathrm{UAV}}+\mathbf{V}_{\text {wind }}
$$

where $\mathbf{V}_{\text {UAV }}$ and $\mathbf{V}_{\text {wind }}$ are the UAV air velocity and wind velocity vectors, respectively. The size for the Warning Zone is denoted as $d_{\text {ahead }}$ and can be expressed as

$$
d_{\text {ahead }}=\left|\mathbf{V}_{\text {total }}\right|\left(t_{\text {update }}\right)
$$

where $t_{\text {update }}$ is the time duration between two consecutive updates of the onboard positioning system. The operational safety bound length $L_{h 1}$ for this 1-dimensional case can be expressed as

$$
L_{h 1}=d_{\text {stop }}+d_{\text {ahead }}=\frac{\left|\mathbf{V}_{\text {total }}\right|^{2}}{2(a)}+\left|\mathbf{V}_{\text {total }}\right|\left(t_{\text {update }}\right)
$$

Eq. (4) is a simple deterministic model for the proposed concept of operational safety bound calculation. Various uncertainties exist and a probabilistic model is proposed based on Eq. (4). The key idea is to include parametrized random variables and use their probability density functions to quantify the uncertainties in the UAV operational safety bound.

Uncertainty in many engineering applications (including the current UAV operational safety bound determination) can be classified into two general categories: aleatoric and 
epistemic uncertainty (Der Kiureghian \& Ditlevsen, 2009; Matthies, 2007). Aleatoric uncertainty in the current application refers to the statistical variability of many operational conditions (e.g., UAV speed and wind speed) and system performance (e.g., deceleration of different UAV and positioning system updating frequency). Epistemic uncertainty refers here simply to the measurement error. Parameters in Eqs. (1-4) are treated as random variables, which are assumed to be described by a summation of aleatoric and epistemic uncertainty. Thus, the deterministic Collision Zone size (Eqs. (1-2)) can be rewritten as

$$
\begin{gathered}
d_{\text {stop }}=\frac{\left|\mathbf{V}_{\text {total }}\right|^{2}}{2(a+\varepsilon(a))} \\
\mathbf{V}_{\text {total }}=\mathbf{V}_{\mathrm{UAV}}+\varepsilon\left(\mathbf{V}_{\mathrm{UAV}}\right)+\mathbf{V}_{\text {wind }}+\varepsilon\left(\mathbf{V}_{\text {wind }}\right)
\end{gathered}
$$

where $a, \mathbf{V}_{\mathrm{UAV}}$, and $\mathbf{V}_{\text {wind }}$ are random variables representing aleatoric uncertainties. Each of these random variables has its corresponding epistemic uncertainty corresponding to the measurement noise, e.g., $\varepsilon(a)$, $\varepsilon\left(\mathbf{V}_{\mathrm{UAV}}\right)$, and $\varepsilon\left(\mathbf{V}_{\text {wind }}\right)$, respectively. Distribution types for aleatoric uncertainties ( $a, \mathbf{V}_{\mathrm{UAV}}$, and $\mathbf{V}_{\text {wind }}$ in Eqs. (1-2)) generally vary for different parameters. Epistemic uncertainty variables $\left(\varepsilon(a), \varepsilon\left(\mathbf{V}_{\mathrm{UAV}}\right)\right.$, and $\varepsilon\left(\mathbf{V}_{\text {wind }}\right)$ in Eqs. (1-2)) are all assumed to be independent Gaussian variables in this study. Other distribution types can be used, and the proposed methodology can be still applied. Now, the deterministic model (Eqs. (1-2)) is changed to a probabilistic model (Eqs. (5-6)) by using the concept of aleatoric and epistemic uncertainty quantification. The same concept can be applied to Eqs. (3-4) and the deterministic Warning Zone size (Eq. (3)) can be expressed as

$$
d_{\text {ahead }}=\left|\mathbf{V}_{\text {total }}\right|\left(t_{\text {update }}+\varepsilon\left(t_{\text {update }}\right)\right)+\varepsilon(\text { GPS })
$$

Uniform distribution is used for $\varepsilon(\mathrm{GPS})$ in this study. The probabilistic operational safety bound size can be expressed as

$$
\begin{aligned}
& L_{h 1}=d_{\text {stop }}+d_{\text {ahead }}=\frac{\left|\mathbf{V}_{\text {total }}\right|^{2}}{2(a+\varepsilon(a))} \\
& +\left|\mathbf{V}_{\text {total }}\right|\left(t_{\text {update }}+\varepsilon\left(t_{\text {update }}\right)\right)+\varepsilon(\mathrm{GPS})
\end{aligned}
$$

Monte Carlo simulation can be employed to estimate the distribution of the operational safety bound size once all input random variables are known. In practice, a threshold value with a certain confidence level is usually used for design and risk management purposes. A 99\% confidence bound (corresponding to a failure probability of $1 \%$ ) is used for most numerical examples. To assess their impact, other confidence bounds are being compared with the $99 \%$ confidence bound in Section 3.

\subsection{Two-Dimensional Case}

The above discussion is for a one-dimensional case and it is straightforward to extend the same concept for the twodimensional case. The key concept is to express the aircraft speed and wind speed as vectors and therefore, the operational safety bound becomes a vectorized field. In the above one-dimensional case, all speed components are parallel to the heading direction of the UAV. In contrast, for the two-dimensional case, the wind direction will have an angle with the UAV's heading direction. The proposed method decomposes the wind velocity vector to two orthogonal components: one parallel to the UAV's heading direction and the other perpendicular to the UAV's heading direction.

The proposed operational safety bound concept will become a complex shape for a fully vectorized description and a simplified "stadium shape" is proposed for easy implementation. A similarly shaped operational safety bound has also been used in (Lam et al., 2009). A schematic illustration of this stadium-shaped operational safety bound is shown in Figure 2. The operational safety bound is described by two lengths: $L_{h 2}$ and $L_{p 2}$. Both are based on the same concept of $d_{\text {stop }}$ and $d_{\text {ahead }}$ introduced in the Section 2.1 for the one-dimensional case. $L_{h 2}$ is along the UAV's heading direction and the calculation is the same as the one shown in the one-dimensional case. $L_{p 2}$ is perpendicular to the heading direction of the UAV and the speed component only has the contribution from wind. $L_{h 2}$ and $L_{p 2}$ are given as

$$
\begin{aligned}
& L_{h 2}=\left|\mathbf{V}_{\mathrm{UAV}}+\varepsilon\left(\mathbf{V}_{\mathrm{UAV}}\right)+\mathbf{V}_{\text {wind }_{h}}+\varepsilon\left(\mathbf{V}_{\text {wind }_{h}}\right)\right| \\
& *\left(t_{\text {update }}+\varepsilon\left(t_{\text {update }}\right)\right) \\
& +\frac{\left|\mathbf{V}_{\mathrm{UAV}}+\varepsilon\left(\mathbf{V}_{\mathrm{UAV}}\right)+\mathbf{V}_{\text {wind }_{h}}+\varepsilon\left(\mathbf{V}_{\text {wind }_{h}}\right)\right|^{2}}{2(a+\varepsilon(a))} \\
& \left|\mathbf{V}_{\mathrm{UAV}}+\varepsilon\left(\mathbf{V}_{\mathrm{UAV}}\right)+\mathbf{V}_{\text {wind }_{h}}+\varepsilon\left(\mathbf{V}_{\text {wind }_{h}}\right)\right|
\end{aligned}
$$

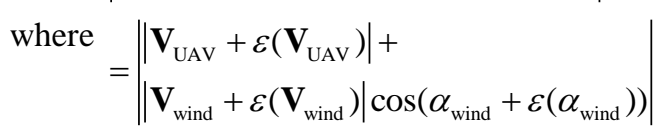$$
L_{p 2}=\left|\mathbf{V}_{\text {wind }_{p}}+\varepsilon\left(\mathbf{V}_{\text {wind }_{p}}\right)\right|\left(t_{\text {update }}+\varepsilon\left(t_{\text {update }}\right)\right)
$$$$
+\frac{\left|\mathbf{V}_{\text {wind }_{p}}+\varepsilon\left(\mathbf{V}_{\text {wind }_{p}}\right)\right|^{2}}{2(a+\varepsilon(a))}+\left|\varepsilon_{G P S}\right|
$$

where

$\left|\mathbf{V}_{\text {wind }_{p}}+\varepsilon\left(\mathbf{V}_{\text {wind }_{p}}\right)\right|=|| \mathbf{V}_{\text {wind }}+\varepsilon\left(\mathbf{V}_{\text {wind }}\right) \mid \sin \left(\alpha_{\text {wind }_{1}}+\varepsilon\left(\alpha_{\text {wind }}\right) \mid\right.$

A new random variable $\alpha_{\text {wind }}$ is introduced in the twodimensional case which is the angle between the wind 
velocity direction and the heading direction of UAV. Monte Carlo simulation and 99\% confidence bound are used for the probabilistic operational safety bound determination. It should be noted that the area of operational safety bound region, $A_{\text {Geofence }}$, is an important metric for future ATC application because it influences the possible density in certain airspace. The operational safety bound area is calculated as

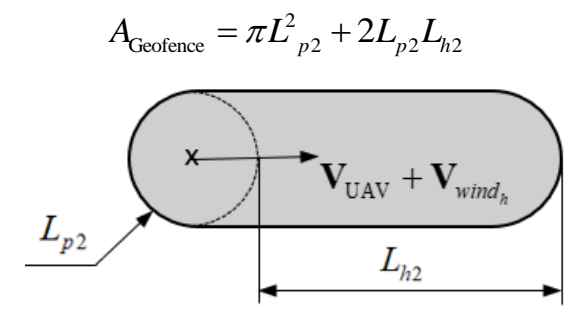

Figure 2. The two-dimensional proposed operational safety bound.

It can be observed that the proposed method has a simple mechanism-based model which includes many types of parameters important for future UTM. The parameters (see Table 1) for the proposed operational safety bound can be classified as the following four major categories: 1) operation, e.g., UAV speed; 2) environment (wind velocity and angle); 3) UAV performance, e.g., maximum deceleration $a$ which is determined by vehicle's motor power, mass, and aerodynamics design; 4) UAV's positioning system and controller, e.g., updating time increment $\left(t_{\text {update }}\right)$.

Table 1. Analysis of parameter type

\begin{tabular}{|l|l|}
\hline Parameter type & Parameter \\
\hline Real-time operation & $\mathbf{V}_{\mathrm{UAV}}$ \\
\hline Environment & $\mathbf{V}_{\text {wind }}, \alpha_{\text {wind }}$ \\
\hline UAV performance & $\left\{\begin{array}{l}\text { Motor power } \\
\text { Mass } \\
\text { Aerodynamics design }\end{array}\right.$ \\
\hline Positioning system & $t_{\text {update }}$ \\
\hline
\end{tabular}

\subsection{Uncertainty Quantification of Model Parameters}

To evaluate the operational safety bound size, data of UAV and environment are needed for the calibration of parameter values and distributions. Many parameters have been reported for various DJI drones (DJI, 2017a, 2017b, 2018), such as the stopping distance. Parameters for different DJI models are shown in Table 2.

Since the UTM concept has not been fully realized to date, distribution information of UAV characteristic is not yet available. Thus, most aleatoric random variables are assumed to be uniform distribution in certain airspace, which can be interpreted as non-informative prior for parameters. Most epistemic random variables are assumed to follow a zeromean Gaussian distribution. The coefficient of variation $(\mathrm{COV})$ is assumed to be 0.05 for all parameter measurement errors. For the measurement noise, zero-mean Gaussian distribution is a good starting point for most measurements of physical quantities unless there is direct evidence otherwise (Weng, Huang, \& Ahuja, 1989). All random variables are assumed to be independent random variables for simplification. A detailed explanation for each random variable is shown below.

It is assumed that the operational speed for UAVs in a certain airspace follows lognormal distribution since speed is always a positive value and UAV tends to fly at its maximum cruise speed which has been set to $20 \mathrm{~m} / \mathrm{s}$ for DJI-like UAVs ("GlobalTelesat"; Guidi, Ricci, Calafate, Gaggi, \& MarquezBarja, 2018). It is assumed that logarithm of the operational speed is normally distributed with a mean of 20 and a standard deviation of 2. In this way, the lower tail of the lognormal distribution can go down to around 14 which is listed in Table 2. The maximum deceleration is assumed to be uniformly distributed between $3.2 \mathrm{~m} / \mathrm{s}^{2}$ and $6.7 \mathrm{~m} / \mathrm{s}^{2}$ based on collected DJI parameters shown in Table 2 (DJI, $2017 \mathrm{a}, 2017 \mathrm{~b}, 2018$ ). The median value of the uniform distribution for $t_{\text {update }}$ is 1 second based on the updating time documented in ("GPS Basics,"; Kingston \& Beard, 2004) and we assume the maximum updating time gap is 2 seconds for most commercially available GPS units. Wind information is generally available for a certain airspace from historical survey data. For example, wind speed varies according to a Weibull distribution with shape parameter $(k)$ ranging from 1.4 to 3 (Justus, Hargraves, \& Yalcin, 1976). The mean value of 2.2 is used in the proposed study. The scale factor $(c)$ in Weibull distribution is closely related to the mean speed $\bar{v}$, since:

$$
\bar{v}=c \Gamma(1+1 / k)
$$

where $\Gamma$ is the gamma function. Average wind speed in Phoenix, AZ is adopted in this study and the value of 2.86 is found in ("2017 Climate Year in Review," 2018). To simplify the sampling of the angle between wind and UAV, it is assumed to follow a uniform distribution ranging from 0 to $2 \pi$ without considering the local wind direction. GPS error is assumed to follow uniform distribution ranging from -1.5 to 1.5 , i.e., $\varepsilon_{\mathrm{GPS}} \sim \mathrm{U}(-1.5,1.5)$. The value of 1.5 is obtained from Mavic 2 Pro/ Zoom drone's user manual (DJI, 2018). A summary for all random variable distributions is shown in Table 3 and they are used for all simulations in this paper. 
Table 2. Data of DJI drones

\begin{tabular}{|l|l|l|l|}
\hline Product & $\begin{array}{l}\text { Max } \\
\text { Velocity } \\
(\mathrm{m} / \mathrm{s})\end{array}$ & $\begin{array}{l}\text { Stop } \\
\text { Distance } \\
(\mathrm{m})\end{array}$ & $\begin{array}{l}\text { Deceleration } \\
\left(\mathrm{m} / \mathrm{s}^{2}\right)\end{array}$ \\
\hline $\begin{array}{l}\text { Mavic 2 } \\
\text { Pro/ Zoom } \\
\text { (DJI, 2018) }\end{array}$ & 20 & 30 & 6.7 \\
\hline $\begin{array}{l}\text { Mavic Pro } \\
\text { (DJI, } \\
\text { 2017a) }\end{array}$ & 18 & 30 & 5.4 \\
\hline $\begin{array}{l}\text { Spark (DJI, } \\
\text { 2017b) }\end{array}$ & 13.9 & 30 & 3.2 \\
\hline
\end{tabular}

Table 3 Distributions to sample parameters

\begin{tabular}{|l|l|l|}
\hline Parameter & $\begin{array}{l}\text { Aleatoric random } \\
\text { variables }\end{array}$ & $\begin{array}{l}\text { Epistemic } \\
\text { random } \\
\text { variables }\end{array}$ \\
\hline $\mathbf{V}_{\mathrm{UAV}}(\mathrm{m} / \mathrm{s})$ & $\operatorname{LogN}(3,0.1)$ & $\mathrm{N}\left(0,0.05 \mathbf{V}_{\mathrm{UAV}}\right)$ \\
\hline$a\left(\mathrm{~m} / \mathrm{s}^{2}\right)$ & $\mathrm{U}(3.2,6.7)$ & $\mathrm{N}(0,0.05 a)$ \\
\hline$t_{\text {update }}(\mathrm{s})$ & $\mathrm{U}(0,2)$ & $\mathrm{N}\left(0,0.05 t_{\text {update }}\right)$ \\
\hline $\mathbf{V}_{\text {wind }}(\mathrm{m} / \mathrm{s})$ & $\mathrm{Weibull}(3.2,2.2)$ & $\mathrm{N}\left(0,0.05 \mathbf{V}_{\text {wind }}\right)$ \\
\hline$\alpha_{\text {wind }}(\mathrm{rad})$ & $\mathrm{U}(0,2 \pi)$ & $\mathrm{N}\left(0,0.05 \alpha_{\text {wind }}\right)$ \\
\hline$\varepsilon_{\mathrm{GPS}}(\mathrm{m})$ & $\mathrm{N} / \mathrm{A}$ & $\mathrm{U}(-1.5,1.5)$ \\
\hline
\end{tabular}

\section{NuMERICAL EXAMPLES IN Two DimENSIONS}

Numerical simulations are shown in this Section to demonstrate the use of the proposed operational safety bound concept. Special focus is on the comparison with two different operational safety bound concepts: static operational safety bound, and dynamic operational safety bound. This Section is organized as follows. First, Monte Carlo simulations are performed to calculate the operational safety bound for the same airspace using different concepts. Fundamental differences in data requirements and results are shown. Secondly, a summary of advantages and disadvantages for each concept is provided based on the performed simulations. Sensitivity analysis is conducted to study the effect of each parameter on the operational safety bound size. Impact of selected risk level on operational safety bound size is also discussed.

\subsection{Numerical Simulation for Different Operational Safety Bound Concept}

As reviewed in Section 1, static operational safety bound and dynamic operational safety bound are two concepts for UAVs. The main difference is that static operational safety bound does not need any knowledge for a specific UAV in the airspace and it directly addresses the total population of UAVs in the airspace (D'Souza et al., 2016; Ong \& Kochenderfer, 2017; Bulusu et al., 2016; Nikolos \& Brintaki, 2005; Weinert et al., 2018; Wu et al., 2018; Bosson \& Lauderdale, 2018; Cook \& Brooks, 2015). Dynamic operational safety bound, on the other hand, includes the velocity information of a specific UAV and wind data for operational safety bound calculation. This greatly reduces the uncertainties of the operational safety bound for a specific UAV and avoids the over-conservative determination of the operational safety bound. The proposed method also belongs to the dynamic operational safety bound concept but includes more information for a specific UAV (such as performance data and positioning system information) compared to the existing dynamic operational safety bound model (D'Souza et al., 2016). Thus, it is expected that the proposed method will further reduce the uncertainties of operational safety bound for a specific UAV. More importantly, the three operational safety bound concepts (e.g., static, dynamic, and the one proposed here) differ fundamentally as the mechanisms to define operational safety bounds. Quantitative assessment of the difference in operational safety bound size is critical to understand their different behaviors, which is the major objective of this Section. Here, we name the static operational safety bound "Static Isotropic (SI) bound", dynamic operational safety bound "Dynamic Isotropic (DI) bound", and the proposed operational safety bound "Dynamic Anisotropic (DA) bound".

\section{Case 1: SI bound}

The horizontal SI bound region is defined to be a circle around a UAV with a radius of static operational safety bound size (D'Souza et al., 2016; Ong \& Kochenderfer, 2017; Bulusu et al., 2016; Nikolos \& Brintaki, 2005; Weinert et al., 2018; Wu et al., 2018; Bosson \& Lauderdale, 2018; Cook \& Brooks, 2015). The radius ( $r$ ) can be calculated as

$$
r=L_{h 2}+L_{p 2}
$$

If taking $1 \%$ as the allowable risk level, the result of the static operational safety bound size based on proposed methodology is identified as the 99th percentile of operational safety bound sizes from direct Monte Carlo simulations. It should be noted that this procedure indicates that all UAVs have the same SI bound size irrespective of their speed or other conditions. Thus, SI bound is a concept focusing on the population behavior rather than individual behavior. Monte Carlo (MC) simulation with 100,000 iterations is performed by randomly sampling each parameter as determined in Table 3. In order to make sure the number 
of MC simulation is sufficient, the percentage error $(E)$ of mean is used to check the accuracy of MC simulation (Driels \& Shin, 2004):

$$
E=\frac{100 Z_{c} \sigma}{\mu \sqrt{n}}
$$

where $n$ is the number of MC samples. $Z_{c}$ is the standard normal distribution $\mathrm{z}$-score such that $c$ is the area under the standard normal curve to the right of $Z_{c}, \sigma$ is the population standard deviation. $\mu$ is mean. At $E$ of $5 \%, c$ is 0.025 and $Z_{c}$ is 1.96. This condition is checked for all MC simulations in this paper to make sure the error of MC simulation is smaller than $5 \%$.

MC simulation results are shown in Figure 3 for $L_{h 2}, L_{p 2}$, and $r$, respectively. Black lines are results from $\mathrm{MC}$ simulations and red dash-lines are best-fitting distributions suggested by Matlab (using fitmethis.m) (Castro, 2018). At the $99 \%$ confidence level, the SI bound size $r$ from simulation is $134.9 \mathrm{~m}$. The area of SI bound is $55990.2 \mathrm{~m}^{2}$. The best-fitting distribution is Gamma distribution with a shape parameter of 8.05 and scale parameter of 8.37. Result of $L_{h 2}$ and $L_{p 2}$ is $130.7,11.5$, respectively. The best-fitting distribution for $L_{h 2}$ is Gamma distribution with a shape parameter of 7.80 and scale parameter of 8.24. Gamma distribution fits $L_{p 2}$ best with a shape parameter of 1.85 and scale parameter of 1.67 .

\section{Case 2: DI bound}

The key concept of DI bound is that the individual UAV information is known and the bound has been developed for this specific UAV rather than for the entire population. Recall that it assumes the circular shape horizontally which does not consider UAV's heading direction (D'Souza et al., 2016).

For instance, parameters are given as follows for a specific UAV

$$
\mathbf{V}_{\mathrm{UAV}}=15 \mathrm{~m} / \mathrm{s}, \quad \mathbf{V}_{\text {wind }}=3 \mathrm{~m} / \mathrm{s}, \alpha_{\text {wind }}=\pi / 4
$$

All other parameters are random variables and are unknown as shown in case 1. 100,000 iterations are conducted. MC simulation results are shown in Figure 4 for $L_{h 2}, L_{p 2}$, and $r$ respectively. The results cannot be directly compared to that for case 1 as SI bound is constant for the entire population considering all possible UAV performance while DI bound is calculated for an individual UAV. We do want to point out that DI bound will have different operational safety bound size for each UAV. For example, in this case, the 99th percentile of DI bound size $r$ is $83.2 \mathrm{~m}$. The area of DI bound is $21746.9 \mathrm{~m}^{2}$. The best-fitting distribution is Generalized Extreme Value (GEV) distribution with a shape parameter of -0.22 , scale parameter of 13.12 and location parameter of 46.41. Result of $L_{h 2}$ and $L_{p 2}$ is 78.0, 6.1, respectively. The best-fitting distribution for $L_{h 2}$ is Generalized Extreme Value (GEV) distribution with a shape parameter of -0.21 , scale parameter of 12.04 and location parameter of 43.40. Weibull distribution fits $L_{p 2}$ best with a shape parameter of 2.79 and scale parameter of 3.77 .

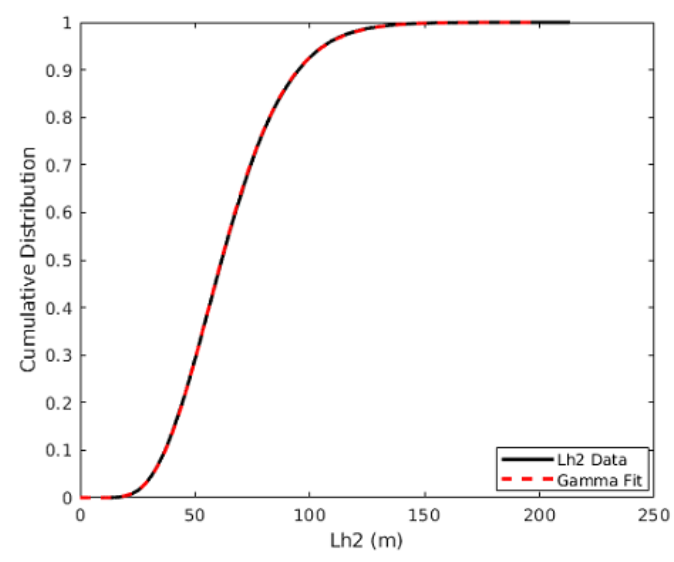

a) Size of $L_{h 2}$

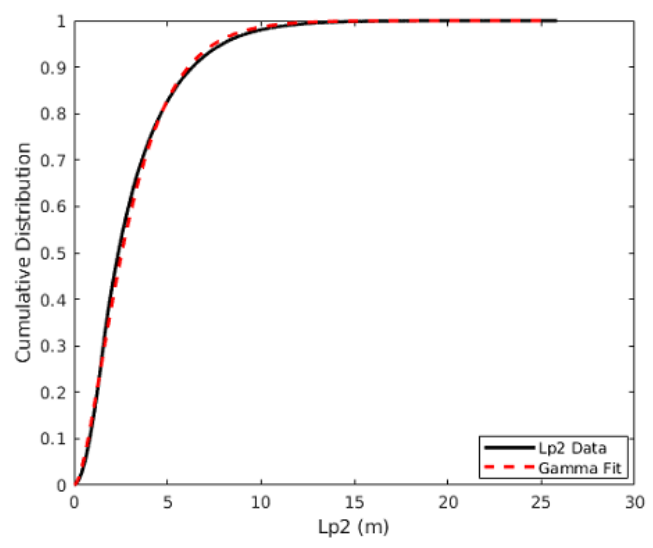

b) Size of $L_{p 2}$

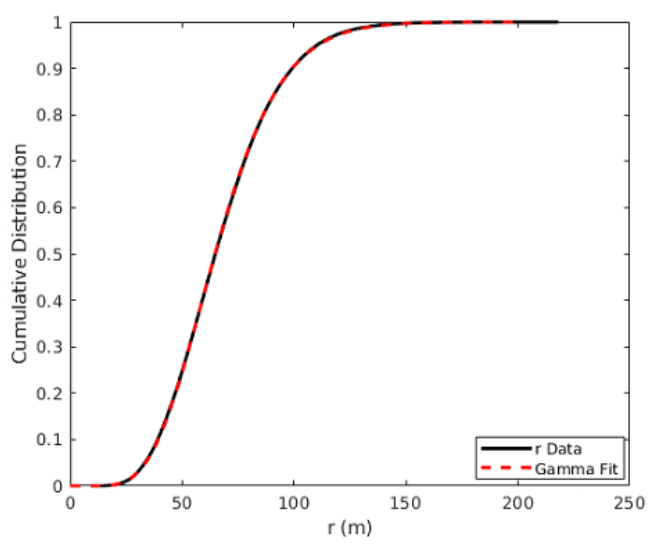

c) Size of $r$

Figure 3. Distribution of SI bound size. 


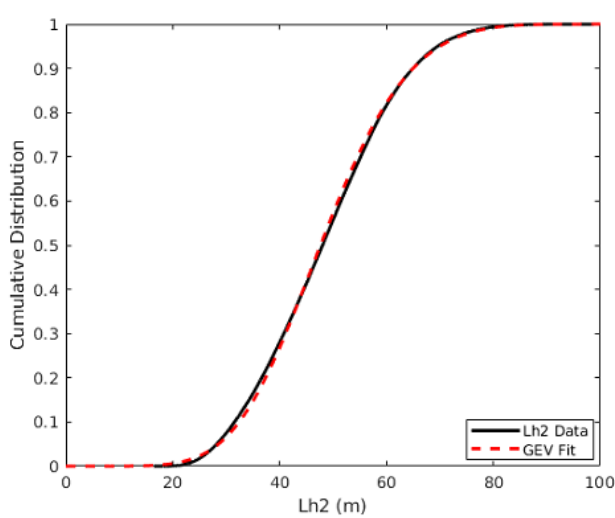

a) Size of $L_{h 2}$

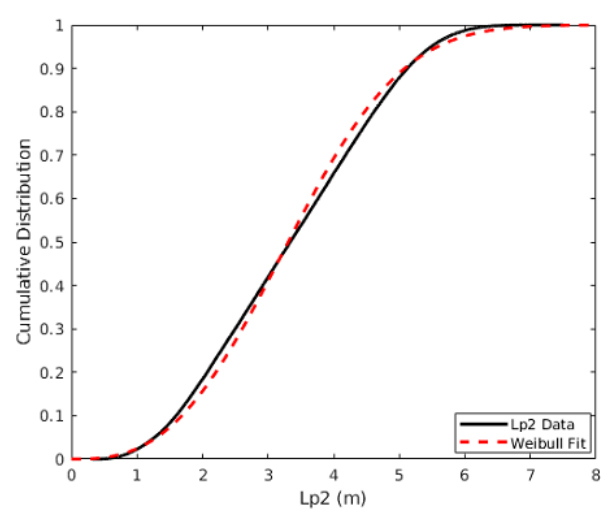

b) Size of $L_{p 2}$

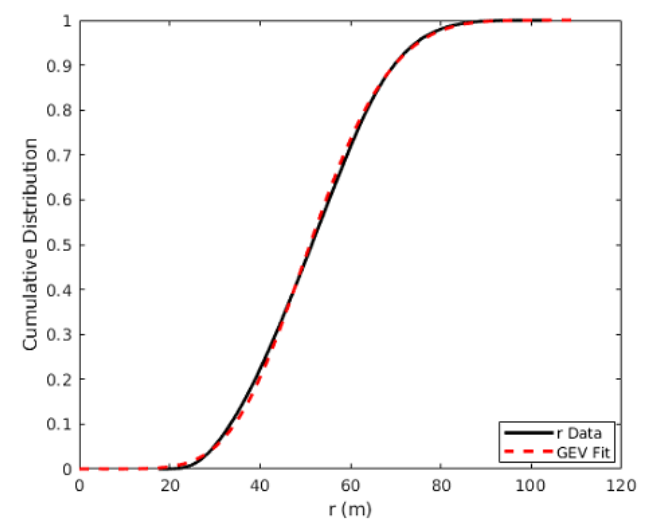

c) Size of $r$

Figure 4. Result of dynamic operational safety bound.

Both $L_{h 2}$ and $L_{p 2}$ are smaller than those in case 1. The reason is that the uncertainty in DI bound is smaller since $\mathbf{V}_{\mathrm{UAV}}, \mathbf{V}_{\text {wind }}$, and $\alpha_{\text {wind }}$ have specific values and are not randomly sampled in the simulations.

\section{Case 3: DA bound}

The proposed method belongs to the dynamic operational safety bound concept applied to an individual UAV. There are two main differences compared with the model in case 2 :
1) it includes more information for an individual UAV (such as deceleration rate and positioning system updating time); 2) operational safety bound shape is vectorized and is not isotropic.

For instance, parameters for a UAV are known for the proposed operational safety bound

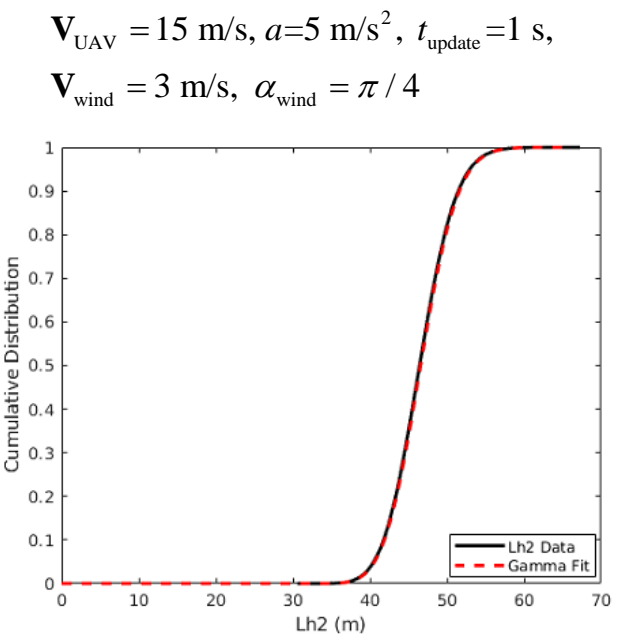

a) Size of $L_{h 2}$

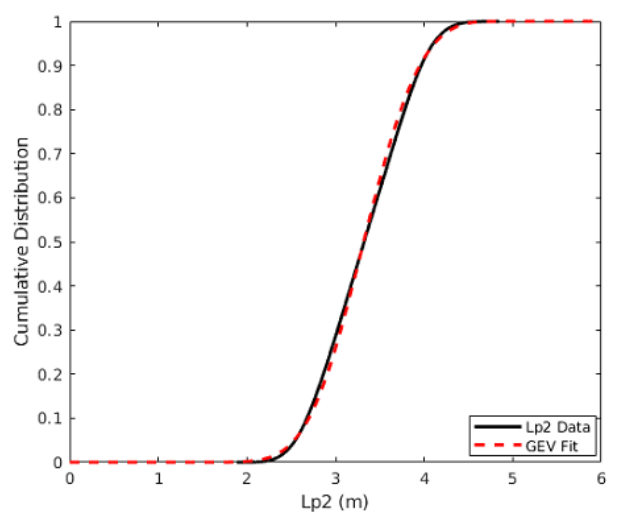

b) Size of $L_{p 2}$

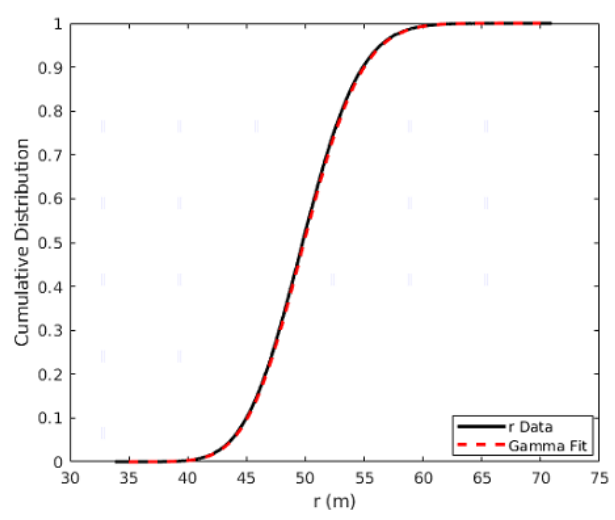

c) Size of $r$

Figure 5. Result of proposed operational safety bound. 
It should be noted that UAV speed, wind speed, and wind angle are the same as those in case 2 for comparison purpose. $100,000 \mathrm{MC}$ simulations have been carried out, and the results are shown in Figure 5. The result of $L_{h 2}$ is $55.8 \mathrm{~m}$ and $L_{p 2}$ is $4.3 \mathrm{~m}$. Using Eq. 11, the area of operational safety bound region is $538.0 \mathrm{~m}^{2}$. The best-fitting distribution for $L_{h 2}$ is the gamma distribution with a shape parameter of 150.9 and a spread parameter of 0.31 . The generalized extreme value distribution fits $L_{p 2}$ best with a shape parameter of -0.28 , scale parameter of 0.48 and location parameter of 3.15 . For $r$, The 99th percentile is $59.3 \mathrm{~m}$. The best-fitting distribution is gamma distribution with a shape parameter of 166.60 and spread parameter of 0.30 .

Both $L_{h 2}$ and $L_{p 2}$ are smaller than those in case 2. The reasons are that the uncertainty in DA bound is smaller since $a$ and $t_{\text {update }}$ have specific values and DA bound takes UAV's heading direction into account. For all three cases, the variance of $L_{h 2}$ is larger than that of $L_{p 2}$. Because the distribution of $L_{p 2}$ depends mainly on $\varepsilon\left(\mathbf{V}_{\text {wind }}\right)$ whose deviation is small due to small wind speed.

From the above study of three cases, it can be seen that under the same risk level, DA bound has the smallest operational safety bound region while SI bound has the largest operational safety bound region. There are mainly two reasons. Firstly, to calculate SI bound, less information is needed which causes larger uncertainty. Secondly, the geometric shape of different bound contributes a lot to the difference of bound size. An anisotropic shape can significantly reduce the area of operational safety bound region. As for the airspace traffic management, smaller operational safety bound can save space and then the airspace has larger capacity and can accommodate more UAVs. Thus, larger operational safety bound is less efficient. Among the three operational safety concepts studied above, DA bound is most efficient and SI bound has lowest efficiency.

\subsection{Comparison of Different Operational Safety Bound Concepts}

Above discussion is for different operational safety bound concepts designed for future UTM operation, including SI bound, DI bound, and DA bound. SI bound is the same size for all UAVs and is very easy to calculate. It is useful especially when there is not enough information, such as UAV performance and weather data. The drawback of SI bound is that it is too conservative since it has the most uncertainty. The efficiency is the lowest which is revealed in section 3.2. Existing DI bound concept does not need information for UAV's deceleration and positioning update frequency compared to DA bound. DA bound is a real-time probabilistic dynamic operational safety bound. It considers UAV's heading direction and is an anisotropic design.
Evaluation of DA bound size needs detailed UAV data and weather data. The advantage of DA bound is the ability to balance safety and efficiency, which shows the highest allowable airspace density among the investigated three operational safety bound concepts. It is preferred for collision avoidance and trajectory plan, especially in future crowded UAS environment.

A summary for the advantages, disadvantages, and suggested application of the three operational safety bound concepts are listed in Table 4.

\subsection{Parameter Sensitivity Analysis}

Sensitivity analysis is conducted for input parameters, $\mathbf{V}_{\mathrm{UAV}}$, $a, t_{\text {update }}, \mathbf{V}_{\text {wind }}$, and $\alpha_{\text {wind }}$. The output variable, $r^{\prime}$ (sum of deterministic $L_{h 2}$ and $L_{p 2}$ ), is used to represent the size of operational safety bound. Finite difference method is used to estimate the sensitivity (i.e., the partial derivatives) for each parameter. The change of each input parameter is set to be $1 \%$ of its mean value for the finite difference estimation (Downing, Gardner, \& Hoffman, 1985). The sensitivity is the ratio of the change in the output variable to the change in the input parameter. Table 5 gives summary statistics of sensitivity analysis.

Table 5 Parameter sensitivity analysis

\begin{tabular}{|l|l|l|l|l|}
\hline Parameter & $\begin{array}{l}\text { Mean } \\
\text { Value }\end{array}$ & $\begin{array}{l}\text { Change } \\
\text { in input }\end{array}$ & $\begin{array}{l}\text { Change } \\
\text { in output }\end{array}$ & $\begin{array}{l}\text { Sensitivity } \\
\text { coefficient }\end{array}$ \\
\hline $\mathbf{V}_{\mathrm{UAV}}(\mathrm{m} / \mathrm{s})$ & 20 & 0.2 & 0.90 & 4.50 \\
\hline$a\left(\mathrm{~m} / \mathrm{s}^{2}\right)$ & 4.95 & 0.0495 & -0.29 & -5.86 \\
\hline$t_{\text {update }}(\mathrm{s})$ & 1 & 0.01 & 0.18 & 18.00 \\
\hline $\mathbf{V}_{\text {wind }}(\mathrm{m} / \mathrm{s})$ & 2.86 & 0.0286 & -0.12 & -4.20 \\
\hline$\alpha_{\text {wind }}(\mathrm{rad})$ & $\pi$ & 0.0314 & 0.10 & 3.18 \\
\hline
\end{tabular}

As shown in Table 5, the position updating time has the strongest effect on the DA bound size. UAV speed and deceleration have slightly higher sensitivity than weather condition, including both wind speed and direction in this study.

\subsection{Impact of Risk Level on Operational Safety Bound Size}

The discussion in Section 3.1 is for demonstration purposes based on a $1 \%$ risk level. In practice, the selected risk level will be determined by regulators and operators. Since UTM is presently still at the conceptual stage, no predefined allowable risk level is available. Thus, a parametric study of the effect of selected risk level on the operational safety 
bound size is performed in this Section. The size based on SI bound, DI bound, and DA bound concept is calculated for risk levels ranging from $10^{-2}$ to $10^{-9}$. Results are shown in Figure 6 where a base-10 log scale is used for the horizontal axis for better visualization. Compared to DI bound and DA bound, the SI bound experiences a significant increase of radius size as a function of risk level. In contrast, the increase of operational safety bound size with respect to the risk level decrease is not significant for DI bound and DA bound concept.

Table 4. Comparison of methods to determine operational safety bound

\begin{tabular}{|c|c|c|c|}
\hline $\begin{array}{c}\text { Operational Safety } \\
\text { Bound Type }\end{array}$ & SI bound & DI bound & DA bound \\
\hline Characteristic & $\begin{array}{l}\text { Circular shape around a } \\
\text { UAV; } \\
\text { Scalar field; } \\
\text { Same size for all UAVs. }\end{array}$ & $\begin{array}{l}\text { Circular shape around a UAV; } \\
\text { Scalar field; } \\
\text { Size depends on UAV } \\
\text { velocity and weather. }\end{array}$ & $\begin{array}{l}\text { Stadium shape around a UAV; } \\
\text { Vector field; } \\
\text { Size depends on UAV velocity, } \\
\text { weather, UAV performance, and } \\
\text { positioning system. }\end{array}$ \\
\hline Pro & $\begin{array}{l}\text { Easy to define avoidance } \\
\text { area; } \\
\text { Needs least information for } \\
\text { real time UAV states. }\end{array}$ & $\begin{array}{l}\text { Only needs information of } \\
\text { UAV velocity and weather to } \\
\text { define avoidance area. }\end{array}$ & $\begin{array}{l}\text { Has least uncertainty; } \\
\text { Highest allowable airspace density } \\
\text { with same risk level. }\end{array}$ \\
\hline Con & $\begin{array}{l}\text { Too conserved; } \\
\text { Not efficient. }\end{array}$ & $\begin{array}{l}\text { Too conserved; } \\
\text { Not efficient. }\end{array}$ & $\begin{array}{l}\text { Needs detailed real-time data; } \\
\text { Not for manual separation. }\end{array}$ \\
\hline $\begin{array}{l}\text { Suggested } \\
\text { Application }\end{array}$ & $\begin{array}{l}\text { Collision detection and } \\
\text { resolution without detailed } \\
\text { information of UAV or } \\
\text { environment. }\end{array}$ & $\begin{array}{l}\text { Collision detection and } \\
\text { resolution without detailed } \\
\text { information of UAV or } \\
\text { environment. }\end{array}$ & $\begin{array}{l}\text { Collision avoidance for a multi- } \\
\text { UAV system; } \\
\text { Trajectory plan especially in } \\
\text { crowded environment. }\end{array}$ \\
\hline
\end{tabular}

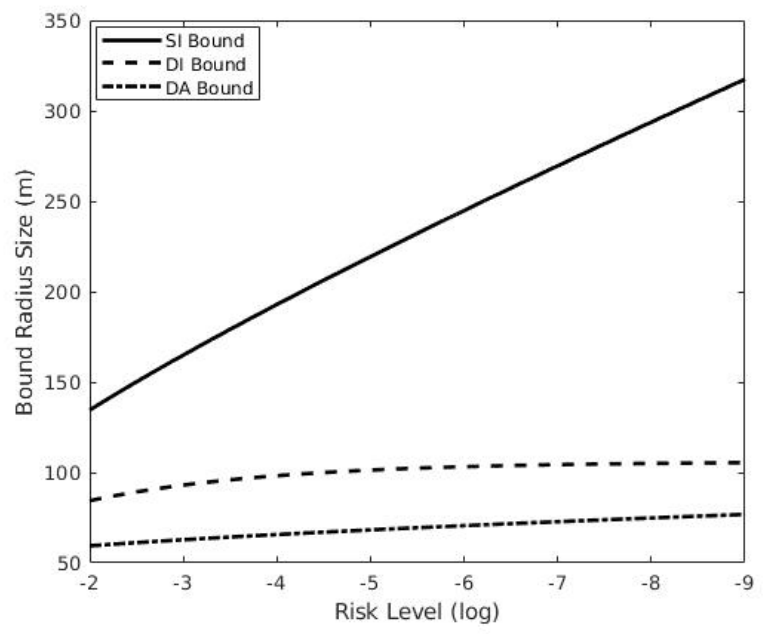

Figure 6. Operational safety bound size under different risk levels

\section{Conclusion}

A new concept of probabilistic dynamic operational safety bound for unmanned aircraft has been proposed in this paper. This operational safety bound is smaller than other safety bounds while maintaining the same risk level. Numerical examples of proposed operational safety bound, static and dynamic operational safety bound are simulated using the Monte Carlo approach. Several major conclusions can be drawn based on the proposed study:

- Uncertainty plays a critical role in determining the operational safety bound size with a certain risk level. Significant variability is observed for operational safety bound size using the collected statistics for small size rotary-wing UAVs;

- Static operational safety bounds can be easily calculated, but are overall too conservative for UTM applications;

- To reduce the uncertainty, it would be advantageous to integrate additional information from UAV operation such as UAV speed, wind speed, and direction, UAV maximum deacceleration rate, update frequency of onboard positioning system;

- An isotropic design is not ideal for operational safety bound shape. Instead, a vectorized shape is suggested to enhance the allowable airspace density with the same risk level;

- Sensitivity study shows that the position updating time has the strongest impact on the operational 
safety bound size and weather conditions and UAV performances have similar sensitivity factors;

- $\quad$ Risk-based operational safety bound size increases as the allowable risk level decreases, but the change of size increase appears to be very different for different operational safety bound concepts.

The proposed study is based on a simple model for rotarywing UAV. The use of a higher fidelity model would help to further reduce the uncertainty of the operational safety bound determination. No vertical separation is considered in this paper and additional work is required for a fully $3 \mathrm{D}$ operational safety bound calculation. Many UAVs will also have other types of positioning system (such as vision-based and radar-based), especially in urban settings. Inclusion of multiple positioning system for operational safety bound calculation will need more study following the same concept in this paper. Additionally, the proposed study assumes normal operation. Failures of information sources will affect the operational safety bound determination (such as jamming of GPS signals). Additional theoretical and experimental study is required to address these rare, but critical scenarios. Finally, UAV operations will likely require on-board or ground-based methods for detecting conflicts and resolving them in real time during flight. That will allow higher conflict probabilities and higher density of traffic than those based purely on choosing low conflict risk strategies as studied in the paper.

\section{ACKNOWLEDGEMENT}

The research reported in this paper was supported by funds from NASA University Leadership Initiative program (Contract No. NNX17AJ86A, PI: Yongming Liu, Technical Officer: Kai Goebel and Anupa Bajwa). The support is gratefully acknowledged.

\section{NOMENCLATURE}

$\begin{array}{ll}d_{\text {stop }} & \text { collision zone size } \\ d_{\text {ahead }} & \text { warning zone size } \\ \mathbf{V}_{\text {total }} & \text { ground velocity vector of UAV } \\ \mathbf{V}_{\text {UAV }} & \text { UAV velocity vector } \\ \mathbf{V}_{\text {wind }} & \text { wind velocity vector } \\ \varepsilon(\cdot) & \text { the error in the bracketed variable } \\ a & \text { UAV deceleration } \\ t_{\text {update }} & \text { update time for UAV positioning system } \\ L_{h 1} & \text { length of operational safety bound in } \\ A_{\text {Geofence }} & \begin{array}{l}\text { one-dimensional case } \\ L_{h 2}\end{array} \\ & \begin{array}{l}\text { length of operational safety bound along the } \\ \text { UAV's heading direction in }\end{array} \\ & \text { two-dimensional case }\end{array}$

$$
\begin{array}{ll}
L_{p 2} & \text { length of operational safety bound perpendicular } \\
& \text { to UAV's heading direction } \\
& \text { in two-dimensional case } \\
\alpha_{\text {wind }} & \text { angle between wind and UAV velocity }
\end{array}
$$

\author{
Subscripts \\ $h \quad$ in the direction along UAV's heading direction \\ $p \quad$ in the direction perpendicular to \\ UAV's heading direction
}

\section{REFERENCES}

2017 Climate Year in Review. (2018). Retrieved from https://www.weather.gov/psr/Year_in_Review_2017

Administration, F. A. (2011). Aeronautical information manual. US Department of Transportation Washington, DC.

Bosson, C., \& Lauderdale, T. A. (2018). Simulation evaluations of an autonomous urban air mobility network management and separation service. In 2018 Aviation Technology, Integration, and Operations Conference (p. 3365).

Brandt, A. M., \& Colton, M. B. (2010). Haptic collision avoidance for a remotely operated quadrotor uav in indoor environments. In Systems Man and Cybernetics (SMC), 2010 IEEE International Conference on (pp. 2724-2731). IEEE.

Bulusu, V., Sengupta, R., \& Liu, Z. (2016). Unmanned aviation: To be free or not to be free? In 7 th International Conference on Research in Air Transportation.

Castro, F. de. (2018). fitmethis. Retrieved from https://www.mathworks.com/matlabcentral/fileexchan ge/40167-fitmethis

Cook, S. P., \& Brooks, D. (2015). A Quantitative Metric to Enable Unmanned Aircraft Systems to Remain Well Clear. Air Traffic Control Quarterly, 23(2-3), 137156.

D’Souza, S., Ishihara, A., Nikaido, B., \& Hasseeb, H. (2016). Feasibility of varying geo-fence around an unmanned aircraft operation based on vehicle performance and wind. In Digital Avionics Systems Conference (DASC), 2016 IEEE/AIAA 35th (pp. 1-10). IEEE.

Der Kiureghian, A., \& Ditlevsen, O. (2009). Aleatory or epistemic? Does it matter? Structural Safety, 31(2), 105-112.

DJI. (2017a). Mavic Pro User Manual.

DJI. (2017b). Spark User Manual.

DJI. (2018). Mavic 2 Pro/Zoon User Manual.

Downing, D. J., Gardner, R. H., \& Hoffman, F. O. (1985). An examination of response-surface methodologies for uncertainty analysis in assessment models. Technometrics, 27(2), 151-163.

Driels, M. R., \& Shin, Y. S. (2004). Determining the number of iterations for Monte Carlo simulations of weapon 
effectiveness. NAVAL POSTGRADUATE SCHOOL MONTEREY CA DEPT OF MECHANICAL AND ASTRONAUTICAL ....

Erzberger, H., Lauderdale, T. A., \& Chu, Y. C. (2012). Automated conflict resolution, arrival management, and weather avoidance for air traffic management. Proceedings of the Institution of Mechanical Engineers, Part G: Journal of Aerospace Engineering, 226(8), 930-949.

Federal Aviation Administration. (2016). FAA Aerospace Forecast: Fiscal Years 2016-2036.

GlobalTelesat. Drones. Retrieved from http://globaltelesat.com/wordpress/drones/

GPS Basics. Retrieved from https://learn.sparkfun.com/tutorials/gps-basics/all

Guidi, B., Ricci, L., Calafate, C., Gaggi, O., \& MarquezBarja, J. (2018). Smart Objects and Technologies for Social Good: Third International Conference, GOODTECHS 2017, Pisa, Italy, November 29-30, 2017, Proceedings (Vol. 233). Springer.

ICAO. (2016). ICAO Doc 4444: Procedures for Air navigation Services (Vol. 4444). Retrieved from http://flightservicebureau.org/wpcontent/uploads/2017/03/ICAO-Doc4444-Pans-Atm16thEdition-2016-OPSGROUP.pdf

International Civil Aviation Organization. (2011). Unmanned Aircraft Systems (UAS). Cir 328 AN/190 (Vol. Cir 328). https://doi.org/10.1016/B978-0-12374518-7.00016-X

Justus, C. G., Hargraves, W. R., \& Yalcin, A. (1976). Nationwide assessment of potential output from windpowered generators. Journal of Applied Meteorology, 15(7), 673-678.

Kingston, D., \& Beard, R. (2004). Real-time attitude and position estimation for small UAVs using low-cost sensors. In AIAA 3rd" Unmanned Unlimited" Technical Conference, Workshop and Exhibit (p. 6488).

Kopardekar, P., Rios, J., Prevot, T., Johnson, M., Jung, J., \& Robinson, J. (2016). Unmanned aircraft system traffic management (utm) concept of operations. In AIAA Aviation Forum.

Lam, T. M., Boschloo, H. W., Mulder, M., \& Van Paassen, M. M. (2009). Artificial force field for haptic feedback in UAV teleoperation. IEEE Transactions on Systems, Man, and Cybernetics-Part A: Systems and Humans, 39(6), 1316-1330.

Liu, Y., \& Goebel, K. (2018). Information Fusion for National Airspace System Prognostics. In PHM Society Conference (Vol. 10).

Matthies, H. G. (2007). Quantifying uncertainty: modern computational representation of probability and applications. In Extreme man-made and natural hazards in dynamics of structures (pp. 105-135). Springer.

Miraglia, G., \& Hook, L. (2017). Dynamic geo-fence assurance and recovery for nonholonomic autonomous aerial vehicles. AIAA/IEEE Digital Avionics Systems Conference - Proceedings, 2017-Septe. https://doi.org/10.1109/DASC.2017.8102088

Nikolos, I. K., \& Brintaki, A. N. (2005). Coordinated UAV path planning using differential evolution. In Intelligent Control, 2005. Proceedings of the 2005 IEEE International Symposium on, Mediterrean Conference on Control and Automation (pp. 549-556). IEEE.

Ong, H. Y., \& Kochenderfer, M. J. (2017). Markov Decision Process-Based Distributed Conflict Resolution for Drone Air Traffic Management. Journal of Guidance, Control, and Dynamics, 40(1), 69-80. https://doi.org/10.2514/1.G001822

Paielli, R. A., \& Erzberger, H. (2017). Trajectory Specification for Terminal Air Traffic: Pairwise Conflict Detection and Resolution. In 17th AIAA Aviation Technology, Integration, and Operations Conference.

Pang, Y., Yao, H., Hu, J., \& Liu, Y. (2019). A Recurrent Neural Network Approach for Aircraft Trajectory Prediction with Weather Features From Sherlock. In AIAA Aviation 2019 Forum (p. 3413).

Wang, Y., Pang, Y., Liu, Y., Dutta, P., \& Yang, B.-J. (2019). Aircraft Trajectory Prediction and Risk Assessment Using Bayesian Updating. In AIAA Aviation 2019 Forum (p. 2936).

Weinert, A., Campbell, S., Vela, A., Schuldt, D., \& Kurucar, J. (2018). Well-Clear Recommendation for Small Unmanned Aircraft Systems Based on Unmitigated Collision Risk. Journal of Air Transportation, 26(3), 113-122.

Weng, J., Huang, T. S., \& Ahuja, N. (1989). Motion and structure from two perspective views: Algorithms, error analysis, and error estimation. IEEE Transactions on Pattern Analysis and Machine Intelligence, 11(5), 451-476.

Wu, M. G., Cone, A. C., Lee, S., Chen, C., Edwards, M. W., \& Jack, D. P. (2018). Well Clear Trade Study for Unmanned Aircraft System Detect And Avoid with Non-Cooperative Aircraft. In 2018 Aviation Technology, Integration, and Operations Conference (p. 2876).

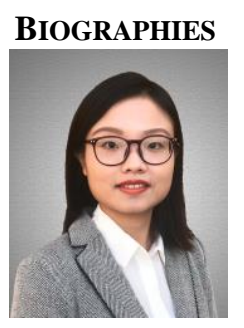

Jueming Hu is a PhD student in the School for Engineering of Matter, Transport \& Energy at Arizona State University. She completed her master at Arizona State University in 2018, and obtained her Bachelors' degree from Southeast University in China in 2017. Her research interests include inverse reinforcement learning and UAS traffic management. 


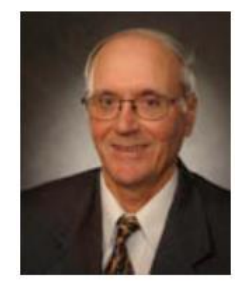

Heinz Erzberger joined Ames Research Center in 1965 after receiving his Ph.D. in electrical engineering from Cornell University. During his career at Ames, he pioneered several concepts for improving the safety and efficiency of aircraft and air traffic control operations. Early in his career, he developed the basic algorithms for fourdimensional guidance and for fuel-optimum flight management. In recent years, he designed the CenterTRACON Automation System (CTAS) to help controllers improve the efficiency and safety of air traffic control. The FAA has deployed the CTAS tool for managing arrival traffic, the Traffic Management Advisor, at major airports in the US. He has published more than 100 papers and has received numerous honors and awards for his research. He holds two patents in the design of air traffic automation tools. $\mathrm{He}$ is a Fellow of the American Institute of Aeronautics and Astronautics (AIAA) and a Fellow of Ames Research Center. $\mathrm{He}$ was awarded the American Society of Mechanical Engineers (ASME) Holley Medal in 2001 and the AIAA's Reed Aeronautical Award in 2004, the AIAA's highest honor for achievements in aeronautical science and engineering. In 2005, he received the Presidential Rank Award for Distinguished Senior Professional from President Bush. Dr. Erzberger was inducted into the Ames Hall of Fame in 2008. He was elected to the National Academy of Engineering of the United States in 2010. Recently, Dr. Erzberger has focused on developing concepts and algorithms for a future air traffic management system that can handle higher traffic levels safely and with increased energy efficiency. After retiring from NASA in February 2006, Dr. Erzberger became an Adjunct Professor at the University of California, Santa Cruz and at Stanford University, where he continued his research in air traffic management with support from several NASA research grants. In early 2010, he returned to NASA Ames as an Intergovernmental Personnel Act (IPA) transferee from UCSC, assuming the position of Senior Advisor in the Aviation Systems Division.

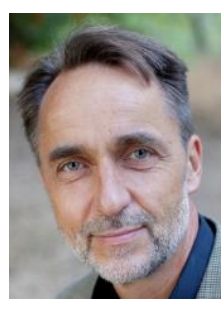

Kai Goebel works at Palo Alto Research Center. His research interest is in the areas of machine learning, real time monitoring for safety, diagnostics, and prognostics. He has fielded numerous applications for manufacturing systems, aircraft engines, unmanned aerial systems, space systems, transportation systems, energy applications, and medical systems. He holds 18 patents and has published more than 300 papers in the field. He received the degree of Diplom-Ingenieur from Technische Universitaet Muenchen in 1990 and the Ph.D. from the University of California at Berkeley in 1996. Dr. Goebel worked between 1997 and 2006 at General Electric's Corporate Research Center in upstate New York where he was also an adjunct professor at Rensselaer Polytechnic Institute. Dr. Goebel is now an adjunct professor at Lulea Technical University. He is a cofounder of the Prognostics and Health Management Society and he is currently associate editor of the International Journal of PHM.

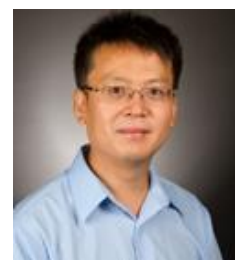

Yongming Liu is a professor in the School for Engineering of Matter, Transport \& Energy at Arizona State University. He completed his $\mathrm{PhD}$ at Vanderbilt University in 2006, and obtained his Bachelors' and Masters' degrees from Tongji University in China in 1999 and 2002, respectively. His research interests include probabilistic methods, diagnostics and prognostics, risk management, materials and structures. He has published over 100 journal articles in the general area of prognostics and health management. He has served on many technical committees in AIAA, ASME, and ASCE. He is an associate fellow of AIAA. 